\title{
Proposal of measures to circumvent soil erosion on flashflood occurrence
}

\author{
V. Bharathan Iyer \\ E.C.E Dept, SCSVMV University, Enathur, Kanchipuram
}

\begin{abstract}
The occurrence of flashflood event is a natural disaster, happening due to excessive pouring of rainfall, creating losses to life and property. Flashflood may be defined as a severe outflow of water, as a sudden occurrence, unlike that of natural flooding events that occur due to heavy rainfall. Their destructivity is comparatively higher than that of the ordinary flooding. One such event that occurred recently in India is discussed here. This paper gives a brief view about its occurrence and clearly analyses the various factors that contributed for its severity. It also focuses about the various steps that can be implemented as a temporary or a permanent state of measures that can prevent losses to life and property.

Keywords: flashflood, deforestation, landslides, afforestation.
\end{abstract}

\section{Introduction}

Flash flood events, through their terms literally refer to sudden outburst of an immediate outflow of water of a huge quantity, flooding areas the water flow travels. The roots for these disastrous occurrences lead to continuous heavy rainfall, which floods the area at a very heavy rate. Statistical report upon the flooding events states that the damages that are caused due to such mishaps accounts to more than 11 trillion $\$$ worldwide. Irrespective of these damages, the floods contaminates the water that flows through the road sides, flowing through sewages, mixing with industrial wastes that contain toxic chemicals and garbage and tend to get mixed with drinking waters. Eventually, it paves way for diseases. Flooding through the mountainous areas may prove more vulnerable than flooding the areas in plains. Since the water level flows from a higher level to that of a lover level, the net kinetic energy of the water flow is heavily increased. The uttarkhand flash flood event that occurred recently is an example for such flashflood occurrence. The entire mountain valley was affected by June 31 , during the height of floods. The prime reasons for this cannot be blamed as a natural disaster. It was a mixture of natural as well as a man made mishap, especially human contribution for this event termed more than the former. Heavy funding and relief measures were deployed to compensate the losses. The government of U.P was funded with $\$ 150$ million initially as a relief measure by the prime minister and other states providing a funding of $\$ 15$ million in total. Other NGOs and youth organizations contributed in a large scale for the rescue missions.

\section{Topographical Features}

The state of uttarkhand lies entirely beneath the Himalayan slope, making it $90 \%$ and the forest makes it to $60 \%$ out of those areas. Since being mountainous, they provide home for variety of flora and fauna that includes snow leopards, tigers and variety of herbs that are possess high medicinal value. The higher levels of the mountainous areas consist of ice caps and rocks. At the range levels between 9,500 and 16,000 ft lay the meadows. Nearing the elevation level of 9,800 to $8,500 \mathrm{ft}$, temperate forests are found. Below 4,900 ft, elevation lie the Himalayan subtropical forests. In addition to this, the upper gangetic plains and the grasslands cover the low lying areas along the entire state. However, for the sake of agricultural purposes, these forests have been cleared gradually.

\section{Situations Before And After Impact}

The impact of the flashflood event happened on $16^{\text {th }}$ July, 2013. Rainfall clouds that were centered over the state of uttarkhand created a sudden outburst, pouring a heavy state of rainfall. The fig 1 is a satellite image taken before the mishap. These images were obtained from DLR aerospace center, Germany, taken trough TerraSAR radar on the spotlight mode of operation. It was estimated approximately that the rainfall occurred was more than $300 \%$ than that of the average rainfall rate of the state of uttarkhand. Such heavy flow of rainfall influenced constant melting of icecaps above the altitude of 3000 meters of Himalayan glaciers, thereby paving way for heavy flow of water. This led to severe outpour of river Mandhakini, creating huge floods at Rudraprayag district, Nepal and gobindghat. 


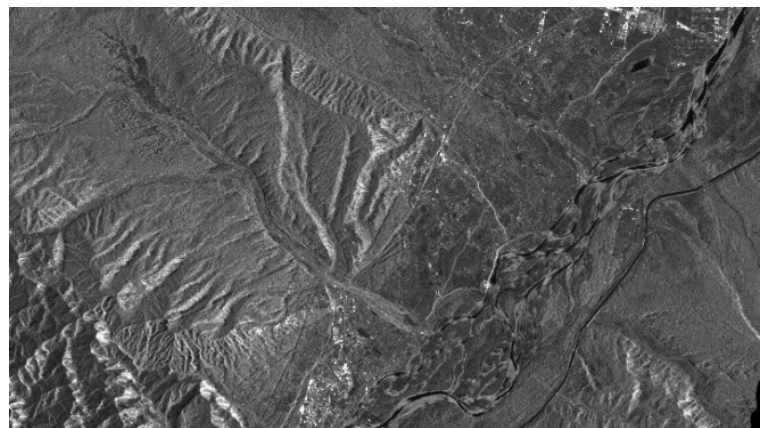

Fig: 1 Areas before the impact. (Courtesy: DLR. Germany)

The flood impact in these areas created destruction was severe. More than 70,000 people were stranded as an immediate effect of flood, out them which $95 \%$ were pilgrims. Nearly 350 houses were destroyed, damaging the transport facilities at many places. Approximately 5000 people were estimated to be the victims of this flashflood event, making it one of the worst impacts after the tsunami incident. More than 2500 people were severely injured and more were indirect targets.

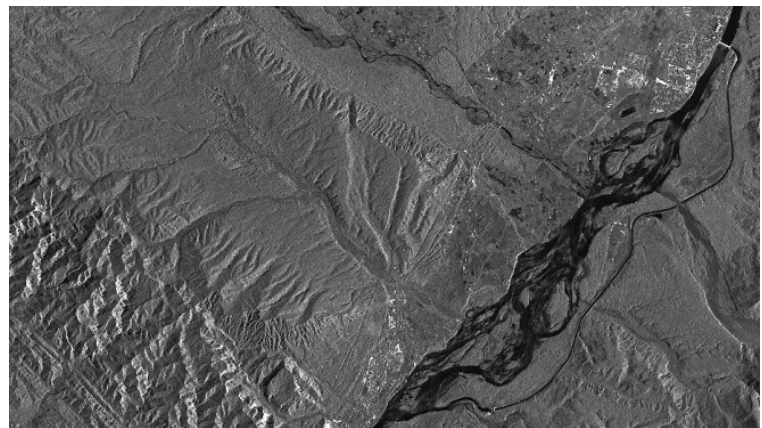

Fig 2: Areas after the impact. (Courtesy: DLR Germany)

Fig 2 shows the places that were severely affected due to the floods. The outpour was more severe when the rivers of Mandhakhini, Alaknanda joined the Ganga creating huge flow. The river banks of Uttarkashi were entirely wiped out by the influence of river Bhagirathi. The impact also paved way for huge landslides, which considerably contributed for the devastation, creating huge obstruction for the transport facilities.

\section{Causes That Termed For The Mishap}

The major cause that is responsible for this impact and huge loss of life and property is ignorance. Construction of buildings and houses in the area that is prone to frequent land slide areas paved way for making the disaster worse. The areas are mountainous steeps that were totally cleared off trees by the localities for the purpose of habitation. Moreover, the trees that present over the landslides were cleared often, for constructional purposes, household and fuel. Such constant clearing of forest directly contributed for deforestation. This paved for the landslide occurrences. The roots of the trees hold the soil particles through heavy flow thereby preventing the soil erosion. Since there weren't trees enough to hold the soil, these eventually lead to losing of bond between the sand particles. Hence, a continuous flow of sand along with the stream flow of water, from the higher level to the lower level eroding the base levels and considerable amount of sand get disposed, leaving the surface level weaker. Moreover, constant rainfall at the Himalayan ranges initiated melting of glaciers to creating huge floods. However, mere rainfall wasn't supposed to be triggering huge flash floods. Estimates describe that the level of rainfall was not that severe enough to produce a devastating flashflood. Various reasons were proposed as causes. But the strongest considered and researched in this paper was that of sudden erosion of soil due to fresh immediate rain pour in considerable quantity greater than that of the average rainfall, enough to trigger landslides of greater extent.

\section{Preventive Measures}

While analyzing the scenario of such flooding events, it comes to the view that preventive measures that are stronger at least enough to reduce the impact level for a temporary period or even at permanent in some cases where of frequent flood occurrence, is mandatory. The causes of these impacts clearly implies that human intervention with nature such as deforestation had contributed a much higher degree, though cannot be accused completely for this disasters. Hence it is necessary of plantation of trees at a much more level in order to prevent 
the soil erosion and landslides during such mishaps, at a greater extent. However, this idea of plantation of trees is not a single-day-output process, as it takes years for a tree to grow to a certain stage in fulfillment of requirements. Hence, it is important to provide an alternate measure to withstand this impact quicker than the former. This paper concentrated on these factors and proposes the following idea as one of the preventive measures that can be implemented in a way that the impact level can be reduced, thereby preventing losses to property and life. Though it mainly concentrates in prevention of landslides that occur due to heavy rainfall, it also focuses on prevention of soil erosion. The schematic view of the proposal the way of implementing them and its characteristics discussed in the upcoming topics.

\section{Proposal Of Implementation}

The idea of implementing this proposal was eyed in concerning the landslide disasters. The landslides occurrence can be rectified by patching the targeted structure with concrete walls. The structure of the target at which, the idea is to be implemented is given in fig 3 as follows.

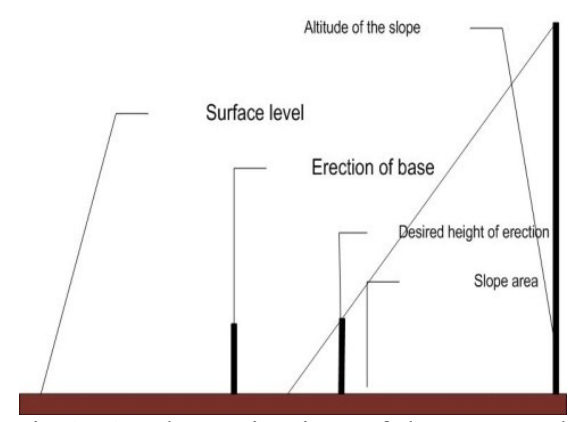

Fig 3: A schematic view of the proposal

The above structure is a steep area, with plantations removed or subjected to deforestation. Since the level of impact is more in such arena, the required level of measure is supposed to begin from its basement levels as follows. The mountainous steep if fixed from its base with initiation of erecting a wall parallel to the face of the steep structure. The wall that is supposed to be erected is made of concrete mixture to avail a firm support to bear enough the load that falls from the upper level during constant rainfall. The basement erection tends to provide a constant holding to the steep by preventing excessive soil to fall as a bulk to the lower level areas. The structure and the construction of the base wall alongside the mountainous steep are explained in the fig 4 as follows.

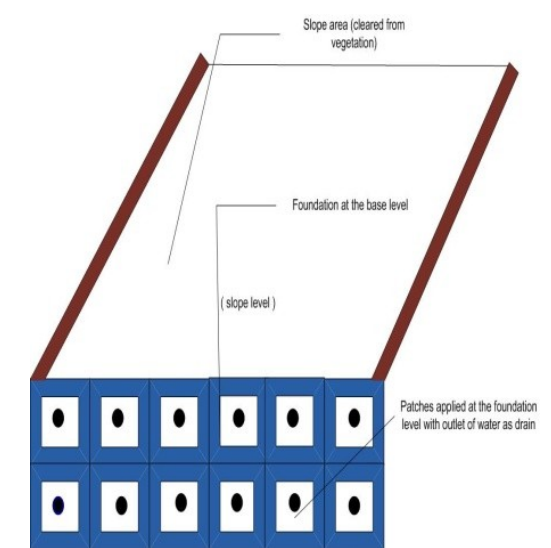

Fig 4: A model of the proposed base patches structure.

From the above figure it is observable that the soil gets stagnated between the walls erected. However, it is subjected to notification that the water also stagnates along side with the mud. Hence it become necessary that to pour out the water from the soil mixture efficiently without creating unnecessary flooding. Hence, the wall structures that are constructed are provided with holes throughout the face side, equispaced. The holes act as a disposure outlet for the water that stagnates between the wall and the steep. Larger the holes, the sand particles too tend to get disposed. Hence, the holes are made smaller the level of tolerance as possible. Since the flow level of water is greater and flexible, the water gushes out from the holes, though much reduced in their speed than before. Such arrangements can prevent the outpour of the mud mixture outside the walls. Though the water is supposed to be disposed through the walls, expulsion of the mud also seems unavoidable at this situation. Hence it is mandatory to arrest the mud outflow to some extent. At this level, plantation of shrubs and bushes 
that have a fibrous root system can be planted over the edges. Since these shrubs grow at a faster rate and have a fibrous root system, the roots can hold the soil particles and avoid the mud flowing out through these outlets. Fig 5 explains the structure of a fibrous root system and its method of implementation.

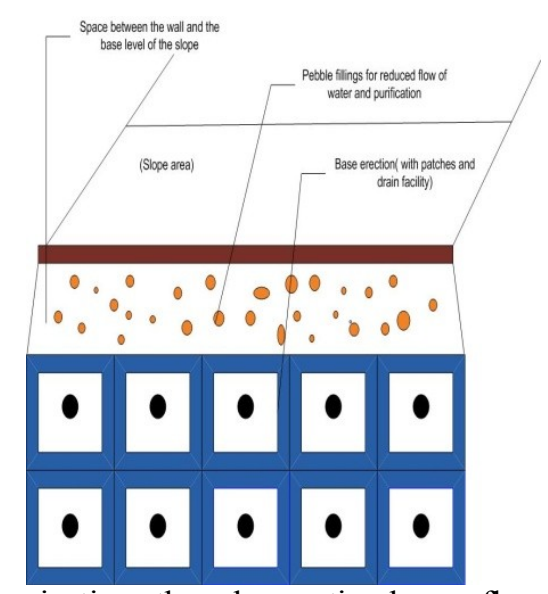

Fig 5: Improvisations though arresting heavy flow by pebbles.

The water that is disposed off through these outlets is not pure. Hence if purified, can also be utilized for various human purposes, such as irrigation and plantation. Pebble stones, which tend to be a natural purifier, can be implemented in this task for purification purpose, filling the interspatial distances between the wall and the mountainous bases. Such arrangement proves very efficient in reducing the speed of water flow. A separate drain is provided for the purpose of water flow so that the flow of water doesn't have a random flow and can be directed in a single, unidirectional way. The drain is designed in a very shallow structure such that they can accommodate more water and placed at a slanting posture for easy flow from upper to that of lower levels. The above methods proposed are cost effective and moreover, the implementation requires a schematic detail. Hence as a precautionary step or immediate restoration of the purpose for a temporary replacement, an alternate solution is also preferred. This method includes spatial filling technique. Initially, the place which is prone to the land slides or the area which is already affected is carefully examined. Then, the amount of soil, which is required to fill up the space, is calculated and taken. Then, the required soil is packed in bags in a large quantity. These bags are intended to fill the landslide areas. The schematic view of arrangement of the bags filled with soil and the methods of filling is described in the following fig 6 .

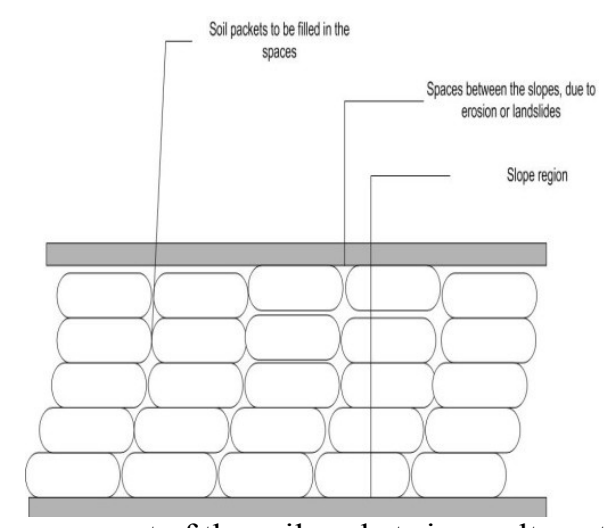

Fig 6: Arrangement of the soil packets in an alternative form.

The soil bags are kept in a position such that they are flat by the surface i.e. horizontally and they are placed in side-by-side alternative manner enough to fix the required place. When the area of filling is more, at this state, a second layer of the bags are placed over the first layer, in the same manner of arrangement. Depending upon the requirement, this procedure is carried out till the concerned area is completely occupied by the soil bags. Moreover, leaving the plain soil in the bags seems more vulnerable, since heavy rains could wash away the soil particles. Therefore, it is necessary to bind the soil particles together to be resistive to soil erosion. Therefore, plants with fibrous root systems are preferred. Consider a stream flow of water, passing through the area that consists of plants with clustered root system. Since fibrous roots are tangled, they arrest the soil within their roots preventing them from getting washed away with the water flow. This principle is implemented in this methodology. The techniques that are used are of two types. They include, 
1. Direct plantation method.

2. Imbibing of seeds.

In the direct method of plantation, the bags that contain the soil are punched with holes. Later, the shrubs are planted through the holes and allowing the upper stem region to directly face the atmosphere. Such condition allows the plant to grow with the help of the sun light. The second method of direct imbibing of seeds includes, applying the seeds directly into the soil sack thereby allowing growth internally. The schematic diagram of the method is given in fig 7 respectively. By seeding the soil in to the sacks, water is not externally sprinkled; rather, these seeds are sown in the wet soil. Such event enables sprouting of the seeds and they tend to grow and expose to the atmosphere with a short span of time. While compared to the former method, the imbibing of seed is very highly efficient in saving the labor and time, since it is need not to be planted or cultivated at any form. Since these two methods doesn't require any specific seasons, they can be implemented at any time of necessity.

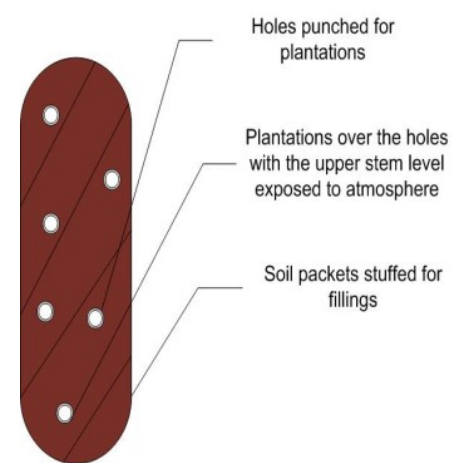

Fig: 7 plantations through the soil packets.

These methods prove to be very advantageous since they are very cost efficient and the content is very less. Since, it doesn't require greater work force, the implementation of the method is less complex and the process becomes easier. The fibrous root system is the key point of this technique in arresting the soil erosion to a greater extent. Lime grass, a species of shrub, has an extensive fiber root system which can accommodate easily to the method. Moreover, the packet arrangement provides a good support and a replacement for the areas of landslides with higher frequency of occurrence. The spatial filling between two slates can achieve better compensation. Moreover, further erosion of soil can be restricted to a greater extent.

\section{Importance Of Afforestation}

Destruction of trees through clearing of forests, for the purpose of cultivation and human habitation creates a sustained ecological imbalance to the ecosystem. These phenomena not only trigger the soil erosion and frequent landslide occurrence, but also enhances poor occurrence of rainfall and induces global warming. This directly contributes to the polar ice melting and rises in the sea levels. This may prove very harmful in the future if proper rectification steps are not taken immediately. Though the root cause seems very simple, its effects find its seriousness in many aspects. Therefore it is very important to take this issue seriously and create awareness about planting of trees and avoid further destruction to the forests. The forests not only serve to provide rain and prevent global warming rather resides as a home for wild animals and rare species. They are threatened to extinction and few of them are at the brink of being extinct. Moreover, the trees play a prime role in controlling the amount of carbon-di-oxide in atmosphere which in excess amount results in global warming and induces green house effect. So, it is necessary to avoid further deforestation and plantation of more trees, which may not be immediately fruitful, but provides a sustained benefit in the future.

\section{Conclusion}

This paper clearly analyses the causes and effects of soil erosion, provided with a sample event. Various methodologies have been proposed in this paper to prevent the soil erosion thereby avoiding its major impact, i.e. the landslides. Landslides not only create destruction to the life and property, but also indicate ecological imbalances. These imbalances are not only a natural defect but also created by humankind through deforestation in form of habitation and urbanization in a larger extent. Even though these things are unavoidable, for the sake of improvisation of lifestyle and comfort, it is important that ecological imbalances may have bad imprecations upon the living organisms present in the Earth that ultimately leads to extinction in a gradual rate. Hence, it is advisable to prevent deforestation to the maximum extent and encourage cultivation of trees at the initial stages, to create a maximum balance to the environment as much as possible rather than discovering solutions to face the impact aftermath. 


\section{Acknowledgments}

I am extremely grateful to Dr. Sankaranarayanan for his valuable guidance throughout this paper work.

\section{References}

[1]. DIR, aerospace centre, Germany.

[2]. Heavy Rainfall Monitoring by Polarimetric C-Band Weather Radars Roberto Cremonini * and Renzo Bechini.

[3]. Doviak, R.J.; Zrnic, D.S. Doppler Radar and Weather Observations, 2nd ed.; Academic Press: New York, NY, USA, 1993; p. 228.

[4]. Zawadzki, I. Factors affecting the precision of radar measurements of rain. In Proceedings of Preprints of the 22nd Conference on Radar Meteorology; American Meteorological Society: Boston, MA, USA, 1984.

[5]. M L Imhoff, "Radar backscatter and biomass saturation -ramifications for global biomass inventory", IEEE Trans. Geosci. Remote Sensing, 1995, 33(2): 511-518

[6]. Cloude, S. R., (1992). Uniqueness of Target Decomposition Theorems in Radar Polarimetry. Direct and Inverse Methods in Radar Polarimetry, Part 1, W.-M. Boener et al. (eds.), pp 267-296. 